\section{Alternative oblique head CT scanning technique reduces bone artifact and improves interpretability of brainstem anatomy}

Sam Kampondeni, ${ }^{1,2}$ Gretchen L. Birbeck, ${ }^{3}$ Robert J. Oostveen, ${ }^{1}$ Colleen Hammond, ${ }^{1}$ Michael J. Potchen ${ }^{1}$

'Michigan State University, Department of Radiology, East Lansing, MI, USA; ${ }^{2}$ Queen Elizabeth Central Hospital, Blantyre, Malawi;

${ }^{3}$ Michigan State University, International Neurologic \& Psychiatric Epidemiology Program (INPEP), East Lansing, MI, USA

\section{Abstract}

Brainstem pathology due to infections, infarcts and tumors are common in developing countries, but neuroimaging technology in these resource-poor settings is often limited to single slice, and occasionally spiral, CT. Unlike multislice CT and MRI, single slice and spiral CT are compromised by bone artifacts in the posterior fossa due to the dense petrous bones, often making imaging of the brainstem nondiagnostic. With appropriate head positioning, the petrous ridges can be avoided with $40^{\circ}$ sagittal oblique scans parallel to either petrous ridge. We describe an alternative sagittal oblique scanning technique that significantly reduces brainstem CT artifacts thereby improving clarity of anatomy. With Institutional Ethical approval, 13 adult patients were enrolled (5 males; 39\%). All patients had routine axial brain CT and sagittal oblique scans with no lesions found. Images were read by 2 readers who gave a score for amount of artefact and clarity of structures in the posterior fossa. The mean artifact score was higher for routine axial images compared to sagittal oblique (2.92 vs. $1.23 ; \mathrm{P}<0.0001)$. The mean anatomical certainty scores for the brainstem were significantly better in the sagittal oblique views compared to routine axial (1.23 vs. 2.77 ; $\mathrm{P}<0.0001)$. No difference was found between the two techniques with respect to the fourth ventricle or the cerebellum (axial vs. sag oblique: 1.15 vs. $1.27 ; \mathrm{P}=0.37$ ). When using single slice CT, the sagittal oblique scanning technique is valuable in improving clarity of anatomy in the brainstem if axial images are non-diagnostic due to bone artifacts.

\section{Introduction}

Although multislice CT and MRI are now widely available in the developed world, single slice, and occasionally spiral, CT remains the neuro-imaging modality available in resourcepoor settings. ${ }^{1,2}$ Unfortunately, single slice CT is compromised by degrading artifacts in the posterior fossa that may render the images non-diagnostic. ${ }^{3-7} \mathrm{CT}$ artifacts may be reduced by avoidance of the artifact-forming bones from the scan planes using appropriate patient positioning. ${ }^{3}$ In the posterior fossa, this may be achieved by avoiding the dense petrous bones and the internal occipital protuberance using $40^{\circ}$ sagittal oblique scans parallel to either petrous bone. Positioning of the patient for this sagittal oblique technique is illustrated in Figure 1. This alternative sagittal oblique CT scanning technique for improved visualization of the brainstem in select patients is routinely employed at the Queen Elizabeth Central Hospital, Blantyre, Malawi. The aim of this paper is to describe this technique and report findings of a study formally comparing posterior fossa image quality using traditional axial versus alternative sagittal oblique techniques on a single slice CT scanner.

\section{Materials and Methods}

This study was conducted at the Queen Elizabeth Central Hospital (QECH), Blantyre, Malawi. Local Ethical Committee approval from the Malawi College of Medicine Research Ethics Committee was obtained for the study. All patients were scanned on a Philips single slice CT scanner, Tomoscan EG (Philips Medical Systems, Netherlands).

\section{Patient population}

Inclusion criteria: adult patients imaged for clinical indications between $1^{\text {st }}$ Feb 2008 and $28^{\text {th }}$ Feb 2008, whose axial scans had artifacts affecting image interpretation as assessed by the QECH radiologist (SK). Only those with normal findings per both axial and sagittal oblique scans were included in the study.

\section{Imaging methods}

All patients underwent traditional routine axial brain CT scanning as follows. Patient position-supine; scan protocol: $120 \mathrm{kVp}, 100$ $\mathrm{mAs}$, slice thickness $5 \mathrm{~mm}$ in the posterior fossa and $10 \mathrm{~mm}$ in the rest of the brain, reconstruction algorithm: standard; scans parallel to the orbito-meatal line. The alternative sagittal oblique scans were carried out as follows. Patient position: prone, head turned $50^{\circ}$ to the right, mid-sagittal plane of the head ver-
Correspondence: Gretchen L. Birbeck, \#324 West Fee Hall, East Lansing, MI 48824, USA.

E-mail: birbeck@msu.edu

Key words: artifact, single slice CT, sagittal oblique, brainstem, brain CT.

Acknowledgments: thanks to $\mathrm{G}$ Msumba and $\mathrm{H}$ Ntutha at Queen Elizabeth Central Hospital for their fine technological support of this work.

Contributions: SK developed method for oblique imaging, directed data collection, reviewed images for clinical purposes and wrote first draft of the paper; GLB developed study design to quantitatively assess value of sagittal oblique CTs, conducted data analysis and assisted in manuscript edits; R0 co-developed training for reviewing oblique images, provided independent reviews with ratings and assisted in manuscript edits; $\mathrm{CH}$ developed images for training in reading of oblique images, assisted with graphics development and manuscript edits; MP co-developed training for reviewing oblique images, provided independent reviews with ratings, assisted in developing method for ratings, developed images for training in reading of oblique images, assisted with graphics development and manuscript edits. All authors approved final draft of manuscript.

Funding: provided by US NIH K23NS046086 (GB).

Conflict of interest: the authors report no conflicts of interest. Ethical approval was given by the Malawi College of Medicine Research Ethics Committee.

Received for publication: 12 May 2010.

Revision received: 7 June 2010.

Accepted for publication: 9 June 2010.

This work is licensed under a Creative Commons Attribution 3.0 License (by-nc 3.0).

CC Copyright S. Kampondeni et al., 2010

Licensee PAGEPress, Italy

Neurology International 2010; 2:e14

doi:10.4081/ni.2010.e14

tical; scan protocol: gantry angulation $=0^{\circ}, 120$ $\mathrm{kVp}, 100 \mathrm{mAs}$, slice thickness $5 \mathrm{~mm}$, spacing 5 $\mathrm{mm}$, reconstruction algorithm: standard; scans done from the left petrous ridge across the whole posterior fossa. Images were printed on radiographic films for clinical review with physical images scanned and electronic versions used for research quantification.

\section{CT interpretation}

The two techniques (traditional routine axial vs. alternative sagittal oblique) were compared as follows: i) artifact severity was scored from 1 to 3 where, $3=$ artifacts render image unusable; $2=$ artifacts may affect reading of film; $1=$ no artifacts; ii) the level of con- 
fidence the radiologist had in visualizing the brainstem, $4^{\text {th }}$ ventricle and cerebellar hemispheres were assigned scores ranging from 13 where: $3=$ total lack of confidence in visualizing a structure; $2=$ reduced level of confidence in visualizing a structure; and $1=$ full confidence in visualizing a structure.

Images were interpreted by 2 readers, MP (neuroradiologist) and RO (general radiologist). The readers underwent orientation training to facilitate interpretation using correlations of sagittal oblique CT images and 3D MRI scans Figure 2 (see Training Manual; Appendix Figures 1-6). A standard set of images depicting posterior fossa anatomy in the sagittal oblique plane is provided (main text Figure 3).

\section{Analysis}

Artifact and anatomical certainty scores were entered into Microsoft Office Excel 2007 before importation into EPI INFO for analysis. Mean artifact scores and anatomical certainty scores for the traditional routine axial versus alternative sagittal oblique views were compared using Student's t-test, unless population variance was significant (per Bartlett's Test), in which case the Mann-Whitney/Wilcoxon's two-sample test was used.

\section{Results}

Thirteen patients met inclusion criteria: age 28-66 years (mean 38.3). Five (39\%) were males. The mean artifact score was higher for routine axial images compared to sagittal oblique (2.92 vs. $1.23 ; \mathrm{P}<0.0001)$. The mean anatomical certainty score for the brainstem were significantly better in the sagittal oblique views versus routine axial (1.23 vs. 2.77 ; $\mathrm{P}<0.0001)$. Reviewers indicated no uncertainty (all scores=1) for visualization of the 4 th ventricle using both the routine axial and the sagittal oblique views. Anatomical certainty scores for the cerebellum was high overall and did not differ in routine axial versus sagittal oblique views (1.15 vs. $1.27 ; \mathrm{P}=0.37$ ).

\section{Discussion}

Where single slice CT is the imaging technology available and axial images are nondiagnostic due to artifacts, the alternative sagittal oblique scanning technique described here adds significant value in terms of increasing the radiologists' ability to discern normal anatomy. The brainstem can be evaluated directly in the sagittal oblique planes or with axial reformatted images for ease of familiarity and to allow left/right comparison

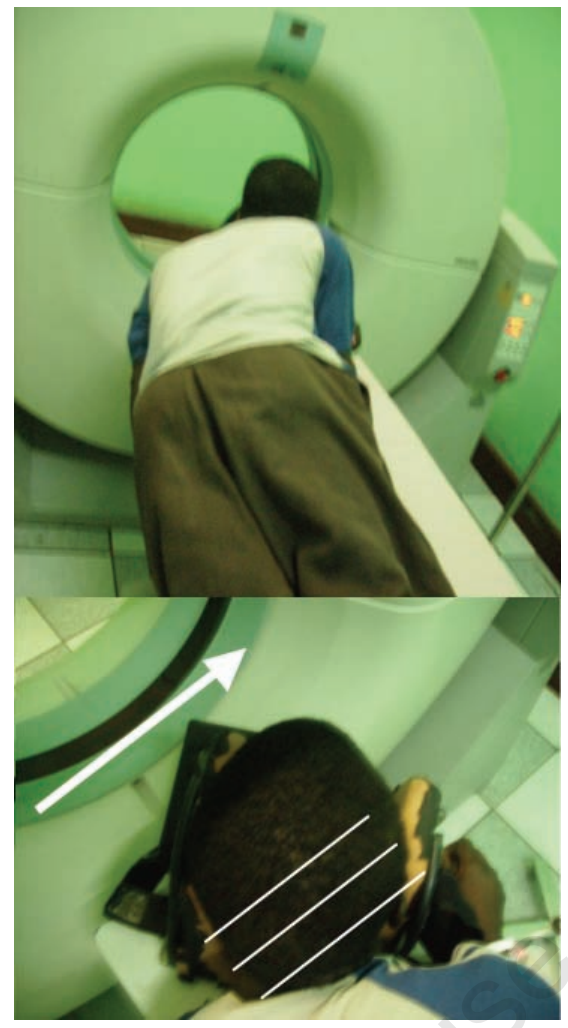

Figure 1. Patient position for obtaining sagittal oblique image. The patient is positioned prone with the mid-sagittal plane of the head vertical and the face turned $50^{\circ}$ to the right. After studying 100 normal adult brain CTs we established that the petrous ridges are anatomically at $50^{\circ}$ to the coronal plane or $40^{\circ}$ to the sagittal plane ( $S$ Kampondeni, oral communication, 2008). This brings the left petrous ridge parallel to the $\mathrm{X}$-ray beam in the CT gantry. The photographs show patient positioning and direction of scans. Reproducibility in the sagittal oblique scanning technique relies on accurate patient positioning: head angulation of 40 degrees to the sagittal plane, keeping the midsgittal plane of the head vertical and maintaining a gantry angulation of 0 .

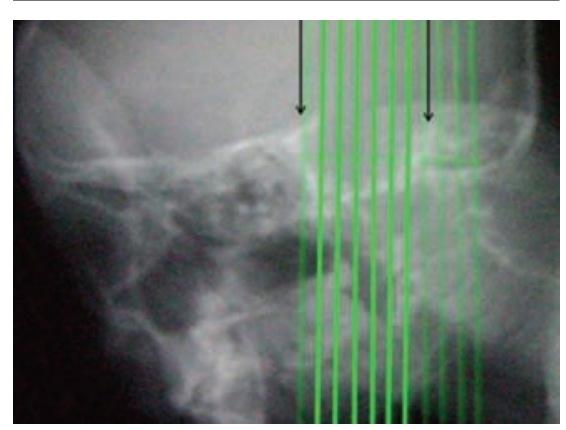

Figure 2. Scanogram for oblique sagittal CT. The scanogram shows study scan lines covering the posterior fossa (arrows show the left and right petrous ridges). Gantry angulation $=0^{\circ}$. Scans are centered at the level of the petrous ridges. Exposure parameters: 120 $\mathrm{kVp}, 100 \mathrm{mAs}$, slice thickness $5 \mathrm{~mm}$, spacing $5 \mathrm{~mm}$. Algorithm: standard. In practice, scans are carried out only across the interpetrous region, from one petrous ridge to the other.

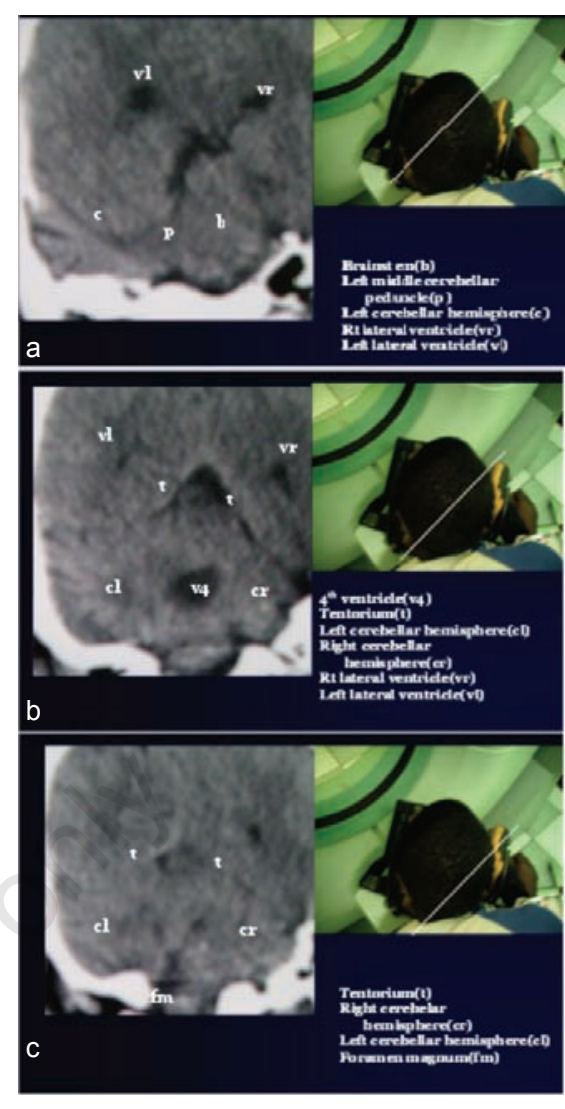

Figure 3. Anatomy of the posterior fossa by $40^{\circ}$ sagittal oblique scans. (a) Scan through the brainstem. (b) Scan through the $4^{\text {th }}$ ventricle. (c) Scan through the cerebellar hemispheres behind the 4 th ventricle.
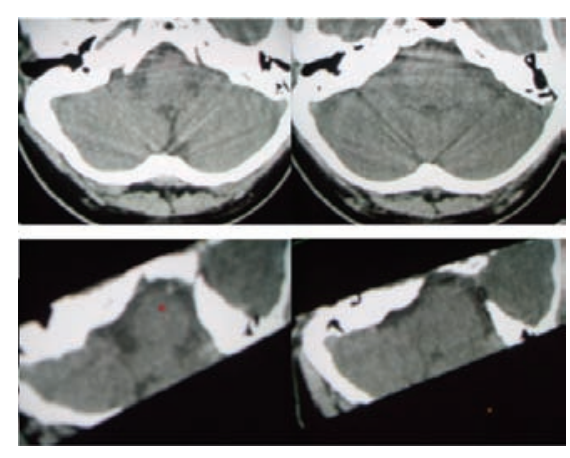

Figure 4. Improved clarity of brainstem anatomy with sagittal oblique technique on axial reformatted images on a spiral CT scanner (GE Synergy, USÄ). Top row: using $2 \mathrm{~mm}$ thin slices (the thinnest possible collimation on this spiral CT scanner, with 2 $\mathrm{mm}$ thin reconstructions, the lowest on this CT scanner) in the posterior fossa, the brainstem and surrounding cisterns are obscured by artifacts. Bottom row: $2 \mathrm{~mm}$ axial reconstructions after acquisition with $2 \mathrm{~mm}$ sagittal oblique scans now show the brainstem, basilar artery and surrounding cisterns with great clarity. All exposure factors were the same in both the spiral-axial and spiral-sagittal oblique techniques: 120 $\mathrm{kVp}, 100 \mathrm{~mA}$, pitch $=1$, table speed 2 $\mathrm{mm} / \mathrm{sec}$, slice thickness $=2 \mathrm{~mm}$. 
within the brainstem (Figure 4). The latter depends upon the availability of MPR (multiplanar reformatting) facility on the CT scanner itself.

The positioning required for this alternative technique may not be safe or feasible in all patients (main text Figure 1). Therefore, the clinician must clinically assess patients prior to such imaging to assure that no airway compromise or other problems occur during the scanning. Further study is needed comparing traditional routine axial versus alternative sagittal oblique views in patients with posterior fossa pathology.

\section{References}

1. Chandramohan A, Eapen A, Govil S, et al. Determining standard liver volume: Assessment of existing formulae in Indian population. Indian $\mathrm{J}$ Gastroenterol 2007;26:22-5.

2. Karabulut N, Ariyürek M. Low Dose CT: Practices and Strategies of radiologists in university hospitals. Diagn Interv Radiol 2006;12:3-8.

3. Barrett JF, Keat N. Artifacts in CT: recognition and avoidance. Radiographics 2004;24:1679-91.

4. Erlt-Wagner B, Eftimov L, Blume J, et al. Cranial CT with 64-, 16-, 4- and singleslice CT systems-comparison of image quality and posterior fossa artifacts in routine brain imaging with standard protocols. European Radiology 2008;18:1720-6.

5. Jones TR, Lane B, Atlas SW, et al. Single versus Multi- detector Row CT of the Brain:Quality Assessment. Radiology 2001;219:750-5.

6. Moström U, Ytterbergh C. Artifacts in computed tomography of the posterior fossa: a comparative phantom study. Journal of computer assisted tomography. 1986;10:560-6.

7. Yeoman LJ, Howarth L, Britten A, et al. Gantry angulation in brain CT: dosage implications, effect on posterior fossa artifacts, and current international practice. Radiology 1992;184:113-6. 